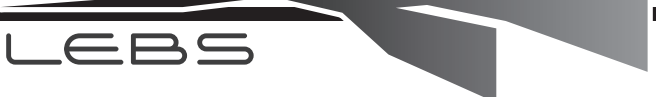

\section{What's in a Smile? Cultural Differences in the Effects of Smiling on Judgments of Trustworthiness}

\author{
Hiroki Ozono ${ }^{1, *}$, Motoki Watabe ${ }^{2}$, \\ Sakiko Yoshikawa', Satoshi Nakashima ${ }^{3}$, \\ Nicholas O. Rule ${ }^{4}$, Nalini Ambady ${ }^{4}$, \\ Reginald B. Adams, Jr. ${ }^{5}$
}

${ }^{1}$ Kokoro Research Center, Kyoto University, Yoshida, Shimoada chi-tyo, Sakyo-ku, Kyoto, 606-8501, Japan

${ }^{2}$ Waseda Institute for Advanced Study, Waseda University, 1-6-1 nishi-waseda, Shinjuku-ku, 169-8050, Japan

${ }^{3}$ Department of Cognitive Psychology in Education, Kyoto Univer sity, Yoshida Hon-machi, Sakyo-ku, Kyoto, 606-8501, Japan

${ }^{4}$ Department of Psychology, Tufts University, 490 Boston Avenue, Medford, Massachusetts, 02155, USA

${ }^{5}$ Department of Psychology, The Pennsylvania State University, 544 Moore Bldg, University Park, PA 16802-3106, USA

"Author for correspondence (h.oozono@p01.mbox.media.kyoto-u. ac.jp)

How do people detect trustworthiness in others? Some researchers have shown that research participants trust smiling faces more than non-smiling faces. We examined cultural differences in this "smile effect." We investigated whether three elements of a smile (smile intensity at the eyes, smile intensity at the mouth, and facial symmetry) would differently influence American and Japanese participants' judgments of trustworthiness. In our experiment, images of 54 American and 69 Japanese male faces were initially rated for intensity of expression in the eyes and mouth, and for symmetry of the smile, by Japanese participants. The images were then presented to 142 American and 80 Japanese participants, who were asked to rate each face's trustworthiness. The results showed that Japanese participants rated as more trustworthy faces with greater upper-half intensity and greater smile symmetry, but rated as less trustworthy faces with greater lower-half intensity. In contrast, American participants rated as more trustworthy faces with greater lower-half intensity, but there was no influence of upper-half smile intensity, nor of smile symmetry.

\section{Keywords}

trustworthiness, smile, cultural differences, symmetry

\section{Introduction}

Accurate detection of trustworthiness is very important for establishing a cooperative relationship between strangers. Such detection allows for the appropriate punishment or exclusion of free riders in order to achieve mutual cooperation among only those participants who are genuinely cooperative (e.g., Frank, 1989).

Previous studies have revealed that humans can detect others' cooperativeness or trustworthiness after brief conversations (Frank, Gilovich, \& Regan, 1993), after watching a short video clip of another person talking (Brown, Palameta, \& Moore, 2003), or after seeing photos taken during the decision moment in a Prisoner's Dilemma game (Verplaeste, Vanneste, \& Braeckman, 2007).

These studies, however, raise the following question: How do people distinguish whether another person can be trusted? Are there certain cues indicating trustworthiness? Smiling is one important candidate for such a cue. Scharlemann, Eckel, Kacelnik, and Wilson (2001) showed that participants trusted smiling partners more than non-smiling partners in a Trust Game.

However, smiling does not always reveal a person's intentions and emotions honestly (Ekman, 1985). In order to achieve precise detection of other's trustworthiness, humans should be able to distinguish an honest smile from a dishonest one. Some studies have shown that there are some features that distinguish an honest smile from a dishonest smile. For example, an honest smile is symmetrical but a dishonest smile is asymmetrical (Gazzaniga \& Smylie, 1990). Also, honest smiles involve the contraction of the orbicularis oculi muscles around the eyes, though dishonest smiles involve only the zygomatic major muscles around the mouth area but not the orbicularis oculi (Ekman, Freisen, \& Davidson, 1990). Based on these findings, there is some evidence that these cues are used specifically to judge trustworthiness (Brown \& Moore, 2002; Krumhuber et al., 2007).

The central question of the present study is whether there are cultural differences in the way specific features of a smile affect judgments of trustworthiness. Eastern and Western cultures have different rules for displaying emotions: Easterners tend to mask or control their emotions while Westerners tend to express their emotions clearly and openly (Matsumoto, Takeuchi, Andayani, Kouznetsova, \& Krupp, 1998). Based on these findings, Yuki, Maddux, and Masuda (2007) hypothesized that Easterners would tend to focus more strongly on the eyes than the mouth when interpreting others' emotions, because the eyes are more difficult to control than the 
Table 1. Multiple Regression of Smile Element Ratings on Personality Trait Ratings by Japanese Participants (Standardized Coefficients)

\begin{tabular}{|c|c|c|c|c|c|}
\hline & Trustworthy & Likeable & Competent & Mature & Dominant \\
\hline $\begin{array}{l}\text { Upper-half smile intensity } \\
\text { (around the eyes) }\end{array}$ & $.21 *$ & $.32 * *$ & .08 & -.10 & -.13 \\
\hline $\begin{array}{l}\text { Lower-half smile intensity } \\
\text { (around the mouth) }\end{array}$ & $-.25^{*}$ & -.04 & $-.37 * *$ & $-.45^{* *}$ & $-.49 * *$ \\
\hline Facial symmetry & $.16+$ & $.21^{*}$ & .04 & -.08 & -.09 \\
\hline $\begin{array}{l}\text { Nationality of the faces } \\
\text { (US }=1 \text {, Japan=2) }\end{array}$ & $-.35^{* *}$ & $-.20+$ & $-.25^{*}$ & $-.23^{*}$ & -.08 \\
\hline
\end{tabular}

$+\mathrm{p}<.10 . * \mathrm{p}<.05 . * * \mathrm{p}<.01$

mouth. On the contrary, Westerners would tend to focus more strongly on the mouth than the eyes, because the mouth is more expressive than the eyes. They conducted two experiments and these hypotheses were confirmed. We believed that this same difference might be present in judgments of trustworthiness, because trustworthiness judgments require accurate inferences of others' genuine emotions and intentions. We also attempted to replicate the findings of Brown and Moore (2002) regarding the influence of smile symmetry on judgments of trustworthiness. Therefore, in the present study, we investigated whether three elements of a smile (smile intensity at the eyes, smile intensity at the mouth, and facial symmetry) would differently influence American and Japanese participants' judgments of trustworthiness. In addition, we investigated the influences of these three elements on four other personality traits: likeability, dominance, maturity and competence.

\section{Method}

\section{Materials}

Fifty-four facial images of candidates for the U.S. Senate in the 2004 election were obtained from a CNN web page (http://www.cnn.com/ ELECTION/2004/pages/results/senate/full.list), and 69 images of candidates for the lower house of the Japanese Diet in the 2000 election were obtained from an online database of political flyers used by candidates (http://politics.j.u-tokyo. ac.jp/data/data $12 . h t m l)$. All candidates were male and all images were grayscale. Images of political candidates were used in this study because it formed part of a larger study, including an investigation into what aspects of faces were correlated with electoral success in American and Japanese national elections (Rule et al., in press).

We first conducted an assessment of the three elements of the smile for each image. Five male and 9 female Japanese undergraduate students rated smile intensity in the upper and lower halves of each face, and another 4 male and 4 female Japanese undergraduate students rated facial symmetry.

For the intensity judgments, participants were shown partial facial images (upper halves separately from lower halves) and were instructed to rate the intensity of each smile on an 8-point
Likert-type scale, where a rating of 0 indicated no smile, a rating of 1 indicated very low intensity, and a rating of 7 indicated very high intensity. Faces were displayed in two blocks, one consisting entirely of upper halves and the other consisting entirely of lower halves. For the symmetry judgments, participants were shown full facial images and were instructed to rate how symmetrical each face was on a 7-point Likert-type scale, where 1 indicated "very asymmetrical" and 7 indicated "very symmetrical." The order of the images was randomized for each participant. Each participant rated all of the images.

The mean rating of smile intensity for upperhalf images was 4.05 ( $\mathrm{SD}=0.83$, range $1.93-6.21$ ). The mean rating of smile intensity for lower-half images was 3.20 ( $\mathrm{SD}=1.16$, range: $1.21-5.57)$. The mean facial symmetry rating was $4.23(\mathrm{SD}=0.63$, range: $2.63-5.88$ ). Therefore, the images used in this study had an appreciable variance on each of these dimensions.

\section{Participants}

One-hundred forty-two American and 80 Japanese undergraduate students participated in the experiment of personality judgments. One-hundred two American participants (65 females, 37 males) rated only American images and the other 40 (23 females, 17 males) rated only Japanese images. Forty Japanese participants (20 females, 20 males) rated only American images and the other 40 (20 females, 20 males) rated only Japanese images.

\section{Procedure and Design}

Participants viewed the facial images on a computer display and were instructed to rate each image for five personality traits. The trials were divided into five blocks. Participants rated one of the five personality traits in each block. At the beginning of each block, participants were instructed as to which trait they would be rating in that block. Each image was presented with the relevant trait at the top of the screen. Participants rated each face for the relevant trait on a 7-point Likert-type scale (e.g., for dominance, a rating of 1 indicated "submissive" and a rating of 7 indicated "dominant"). Block order and the order of images within each block were randomized for each participant. 
Table 2. Multiple Regression of Smile Element Ratings on Personality Trait Ratings by American Participants (Standardized Coefficients)

\begin{tabular}{lcccc}
\hline & Trustworthy & Likeable & Competent & Mature \\
\hline $\begin{array}{l}\text { Upper-half smile intensity } \\
\text { (around the eyes) }\end{array}$ & .10 & $.33^{* *}$ & -.08 & -.10 \\
$\begin{array}{l}\text { Lower-half smile intensity } \\
\text { (around the mouth) }\end{array}$ & $.32 * *$ & $.65^{* *}$ & $-.23^{*}$ & -.12 \\
Facial symmetry & .08 & $.14^{*}$ & .12 & $-.67^{* *}$ \\
$\begin{array}{l}\text { Nationality of the faces } \\
\text { (US=1, Japan=2) }\end{array}$ & $.21+$ & $.25^{* *}$ & $-.23^{*}$ & -.03 \\
\hline
\end{tabular}

$+\mathrm{p}<.10 . * \mathrm{p}<.05 . * * \mathrm{p}<.01$

\section{Results and Discussion}

We performed a series of multiple regression analyses, with nationality of the faces, upper-half smile intensity, lower-half smile intensity, and facial symmetry as the independent variables in each regression model, and personality trait ratings as the dependent variables. Standardized coefficients of regression models for personality trait ratings by Japanese participants are given in Table 1. Those for American participants are given in Table 2.

The results show clear cultural differences for trustworthiness. Japanese participants rated as more trustworthy faces with greater lower-half intensity and greater smile symmetry, but rated as less trustworthy faces with greater upper-half intensity. In contrast, American participants rated as more trustworthy faces with greater lower-half intensity, but there was no influence of upper-half smile intensity, nor of smile symmetry.

Why did this cultural difference occur? It is possible that a similar effect to that found in Yuki et al. (2007) was in operation. In Japanese culture, individuals tend to control their expressions of emotion, so Japanese raters tended to use smile intensity of the eyes and smile symmetry, which is a less controllable feature than smile intensity of the mouth, in order to make judgments about trustworthiness. In addition, smiling is not always appropriate in contexts where people are trying to establish cooperative relationships because expression inhibition is the norm in Japan. Therefore, highly expressive smiles, where the mouth was strongly smiling, were less trusted than by non-expressive ones. On the other hand, in the American culture, people express their emotions and intentions more overtly, so American raters focused on the mouth, which is the most expressive part of the face.

For the other four personality traits, a variety of cultural differences and similarities were found (Tables 1 and 2). These findings are complex, and we cannot explain these comprehensively. However, these findings suggest that people use different elements of a smile in judging different personality traits, and that the elements used for particular judgments is culture-dependent. Moreover, we found an effect of nationality of the stimulus faces. Japanese participants rated American faces as more trustworthy than Japanese ones, while American participants rated Japanese faces as more trustworthy than American ones (Tables 1 and 2). Though these results were not related to the effects found for specific elements of the smile, they are interesting. Also, it should be noted that more detailed analysis revealed an interaction effect between nationality of the faces and smile intensity. The positive effect of upper-half smile intensity for trustworthiness was stronger for American faces than Japanese ones, for both Japanese raters and American raters. It is difficult to explain this finding at this point, and further study is needed.

Our conclusions are subject to some limitations. First, the three elements of the smiles were rated only by Japanese participants. Ratings of smile intensity and symmetry may differ across cultures, so American participants should also rate these elements in the future studies. Second, all of our images were photos taken for the purpose of electoral campaigning. The expressions in each photograph may therefore be different from those seen in daily life. Third, only male faces were used in this experiment. How our findings are applicable to female stimulus faces should be examined in future work.

Although we found cultural differences in raters' inferences of others' trustworthiness, we only examined the receivers of facial signals. To fully investigate the detection of cheaters and cooperators, it would be necessary to study the senders of facial signals. Cultural differences in how people express their intention and emotion when they have incentive to be trusted have yet to be investigated, and the full mechanism underlying inferences of trustworthiness has yet to be revealed. Finally, the origins of these cultural differences, and how these cultural differences have been maintained or changed historically, await future research efforts.

\section{References}

Brown, W. M., \& Moore, C. (2002). Smile asymmetries and reputation as reliable indicators of likelihood to cooperate: An evolutionary analysis. In S. P. Shohov (Ed.), Advances in Psychology Research (Vol. 11, pp. 59-78). New York: Nova Science Publishers.

Brown, W. M., Palameta, B., \& Moore, C. (2003). Are there nonverbal cues to commitment? An exploratory study using the zero-acquaintance video presentation paradigm. Evolutionary 
Psychology, 1, 42-69.

Ekman, P. (1985). Telling lies. New York: Norton.

Ekman, P., Freisen, W. V., \& Davidson, R. J. (1990).

The Duchenne smile - emotional expression and brain physiology. Journal of Personality and Social Psychology, 58, 342-353.

Frank, R. H. (1989). Passions within reasons: The strategic role of emotions. New York: W. W. Norton.

Frank, R. H., Gilovich, T., \& Regan, D. T. (1993). The evolution of one-shot cooperation: an experiment. Ethology and Sociobiology, 14, 247256. (doi:10.1016/0162-3095(93)90020-I)

Gazzaniga, M. S., \& Smylie, C. S. (1990). Hemispheric mechanisms controlling voluntary and spontaneous smiling. Journal of Cognitive Neuroscience, 2, 239-245.(doi:10.1037/00223514.58.2.342)

Krumhuber, E., Manstead, A. S. R., Cosker, D., Marshall, D., Rosin, P. L., \& Kappas, A. (2007). Facial dynamics as indicators of trustworthiness and cooperative behavior. Emotion, 7, 730-735. (doi:10.1037/1528-3542.7.4.730)

Matsumoto, D., Takeuchi, S., Andayani, S., Kouznetsova, N., \& Krupp, D. (1998). The contribution of individualism vs. collectivism to cross-national differences in display rules. Asian Journal of Social Psychology, 1, 147-165. (doi:10.1111/1467-839X.00010)

Rule, N. O., Ambady, N., Adams, R. B., Jr., Ozono, H., Nakashima, S., Yoshikawa, S., \& Watabe, M. (in press). Polling the face: Prediction and consensus across cultures. Journal of Personality and Social Psychology. (doi:10.1037/ a0017673)

Scharlemann, J. P. W., Eckel, C. C., Kacelnik, A., \& Wilson, R. K. (2001). The value of a smile: Game theory with a human face. Journal of Economic Psychology, 22, 617-640. (doi:10.1016/ S0167-4870(01)00059-9)

Verplaetse, J., Vanneste, S., \& Braeckman, J. (2007). You can judge a book by its cover: the sequel. A kernel of truth in predictive cheating detection. Evolution and Human Behavior, 28, 260-271. (doi:10.1016/j.evolhumbehav.2007.04.006)

Yuki, M., Maddux, W. W., \& Masuda, T. (2007). Are the windows to the soul the same in the East and West? Cultural differences in using the eyes and mouth as cues to recognize emotions in Japan and the United States. Journal of Experimental Social Psychology, 43, 303-311. (doi:10.1016/j.jesp.2006.02.004) 